\title{
Dosimetry of thermal neutron beamlines at a pulsed spallation source for application to the irradiation of microelectronics
}

\author{
Carlo Cazzaniga, Davide Raspino, G. Jeff Sykora \\ and Christopher D. Frost
}

\section{Published version information}

Citation: C Cazzaniga et al. 'Dosimetry of thermal neutron beamlines at a pulsed spallation source for application to the irradiation of microelectronics.' IEEE Trans Nucl Sci 68, no. 5 (2021): 921-927.

DOI: $\underline{10.1109 / T N S .2021 .3064681}$

(C)2021 IEEE. Personal use of this material is permitted. Permission from IEEE must be obtained for all other uses, in any current or future media, including reprinting/republishing this material for advertising or promotional purposes, creating new collective works, for resale or redistribution to servers or lists, or reuse of any copyrighted component of this work in other works.

This version is made available in accordance with publisher policies. Please cite only the published version using the reference above. This is the citation assigned by the publisher at the time of issuing the AAM. Please check the publisher's website for any updates. 


\title{
Dosimetry of Thermal Neutron Beamlines at a Pulsed Spallation Source for Application to the Irradiation of Microelectronics
}

\author{
Carlo Cazzaniga, Davide Raspino, G. Jeff Sykora and Christopher D. Frost
}

\begin{abstract}
GEM detectors and activation foils have been used for dosimetry of thermal beamlines of a pulsed neutron source. The first is an active detector that performs measurements using the time of flight technique, taking advantage of the pulsed nature of the source. The same detector has been used successfully to measure the profiles of the beams. The second is a passive irradiation method that independently confirms the measured fluxes of EMMA and ROTAX beamlines of the ISIS neutron source. They feature different thermal spectra, the first being moderated with water $(300 \mathrm{~K})$ and the second with liquid methane $(100 \mathrm{~K})$. These two characterized beamlines have then been used for the irradiation of microelectronics for single event effect testing using reference SRAM module. It is shown that results are consistent, and that a correction factor must be applied to scale the results on the cold beamline to the one at room temperature.
\end{abstract}

Index Terms - thermal neutrons, time of flight, activation foils, single event effects.

\section{INTRODUCTION}

$\mathrm{I}^{\mathrm{n}}$ ndustry and academia are increasingly performing irradiation tests of microelectronics components to study Single Event Effects (SEE) that can disrupt the operation of systems often used in safety-critical applications [1-2]. For terrestrial and avionic environments, spallation facilities are used to reproduce the cosmic-ray induced atmospheric spectrum [3]. This is a white neutron spectrum with energies up to hundreds of $\mathrm{MeV}$ [4]. ChipIr, a beamline at the Rutherford Appleton Laboratory (UK), uses the spallation of $800 \mathrm{MeV}$ protons of the ISIS accelerator on a tungsten target to produce an atmospheric-like neutron beam [5,6]. Other reference facilities for the atmospheric environment are LANSCE [7] in the USA and TRIUMF [8] in Canada.

The atmospheric neutron radiation field, however, presents a very significant and variable component of thermal neutrons [9]. Thermal neutrons are produced by the moderation of fast neutrons in materials. By definition their spectrum comes from a Maxwell-Boltzmann distribution with room temperature at ground level $\mathrm{kT}=$

Carlo Cazzaniga, Davide Raspino, G. Jeff Sykora and Christopher D. Frost are with ISIS Facility, UKRI-STFC, Rutherford Appleton Laboratory, Didcot OX11 0QX, UK. (e-mail: carlo.cazzaniga@stfc.ac.uk)
$25 \mathrm{meV}(\mathrm{T}=300 \mathrm{~K})$. For a composite neutron spectrum, as generated by scattering from a fission or spallation neutron production process, the neutron spectrum is often divided in to a thermal, a 1/E (or epithermal), and a fast component. Thermal neutron fluxes are defined for $\mathrm{E}<$ $0.5 \mathrm{eV}$, which is the threshold of absorption of cadmium (see the nuclear cross section of cadmium in Fig. 1). This definition of thermal neutron fluxes, as being dictated by the cadmium cut-off, is a fully experimental definition related to the need to have a practical measurement method and it is standard practice in nuclear physics. ${ }^{113} \mathrm{Cd}$ has a very large (20,615 barn) resonance absorption cross section which is often used as a cover or attenuating material in dosimetry measurements - see Fig. 1.

SEE are induced by thermal neutrons when boron is present in (or close) to the sensitive volume of microelectronics devices [10]. This is because ${ }^{10} \mathrm{~B}$ has a very high cross section for the $(n, \alpha)$ reaction at thermal energies (see Fig. 1). ${ }^{10} \mathrm{~B}$ is $20 \%$ of natural boron, the rest being ${ }^{11} \mathrm{~B}$. Boron is necessary for the manufacture of modern semiconductors, so many commercial devices contain ${ }^{10} \mathrm{~B}$. It is well known that an easy mitigation solution would be to use enriched ${ }^{11} \mathrm{~B}$. However, isotope enrichment is very expensive and often not used in commercial electronics that are now frequently used in critical applications. Recent studies have shown that in some cases the Failure In Time (FIT) due to thermal neutrons can be comparable to the FIT due to fast neutrons in terrestrial applications $[11,12]$. It is important to notice that sensitivity to thermal neutrons is very device-dependent. The effect is important for devices with a low LET threshold in the SEE cross section, i.e. a low critical LET.

Moreover, the thermal neutron flux can be increased by the presence of moderating material (hydrogenous material). Modern data centres and supercomputing facilities contain large quantities of materials such as concrete structures and floors, thick walls, and water cooling units. These can all potentially increase the flux 
of thermal neutrons, such that they are a major concern for supercomputing applications [13].

Recent studies with $7 \mathrm{~nm}$ bulk FinFET report that the presence of ${ }^{10} \mathrm{~B}$ in the metal stack makes contribution of thermal-neutron-induced SEEs significant at the 7-nm node [14].

These recent studies motivate the need of accelerated SEE testing using thermal neutrons. Pulsed spallation sources can provide thermal neutron beams using moderators [15]. In this work, the dosimetry techniques that have been used to characterize two thermal beamlines are presented. The time of flight (ToF) technique can be utilized [16], making use of the pulsed source to provide the neutron energy spectrum. Use of activation foils is the reference technique used to independently verify the neutron fluence and to infer the flux $[17,18]$.

In the last part of this work, characterization SEE measurements, performed with SRAM memories, are presented. It can be noticed that care must be taken in cases when the temperature of the moderator deviates from room temperature. This is because the nuclear cross section of ${ }^{10} \mathrm{~B}$ is strongly dependent on the neutron energy, as shown in Fig. 1. A correction factor must be calculated to relate the SEE induced by a thermal neutron Maxwell-Boltzmann energy distribution at a temperature other than that of the reference room temperature to the corresponding SEE expected at the reference temperature.

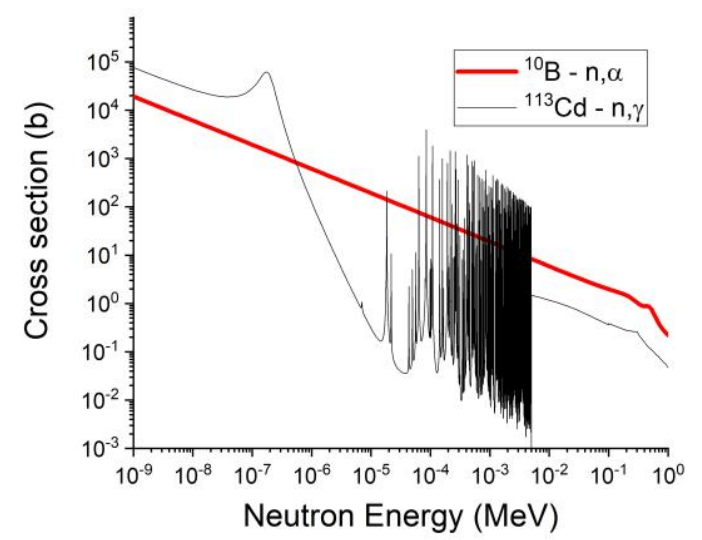

Fig. 1. Nuclear cross sections of interest. The $(\mathrm{n}, \alpha)$ reaction on ${ }^{10} \mathrm{~B}$ is responsible for SEE induced by thermal neutrons. The capture reaction on ${ }^{113} \mathrm{Cd}$ is used for shielding of thermal neutrons with $\mathrm{E}<0.5$ $\mathrm{eV}$.

\section{II.THERMAL NEUTRONS BEAMLINES}

The ISIS pulsed neutron source [19] is based on a synchrotron accelerator that collides $800 \mathrm{MeV}$ protons on tungsten targets (on two target stations) to produce neutrons by spallation. The accelerator is pulsed at $50 \mathrm{~Hz}$, with $40 \mathrm{~Hz}$ on the first target station, and $10 \mathrm{~Hz}$ on the second. The average current is about $200 \mu \mathrm{A}$.

A beryllium reflector is used to maximize the neutron flux on the moderators. In the first target station, fast neutrons are slowed down in four different moderators. Two of them use water at room temperature $(\mathrm{T}=300 \mathrm{~K})$, one uses liquid methane $(\mathrm{T}=100 \mathrm{~K})$ and the fourth consists of liquid hydrogen $(\mathrm{T}=20 \mathrm{~K})$. The different temperatures result in different energy thermal neutron beams. A schematic of the target station is presented in Fig. 2.

In this work, two thermal beamlines of the first target station have been considered, EMMA (previously called HET) [20] and ROTAX [21], that have a line of sight on the water and liquid methane moderators, respectively. The distance from the moderator to the irradiation positions are $16 \mathrm{~m}$ and $12 \mathrm{~m}$, respectively.

On both beamlines, a chopper (a rotating device used to block a portion of the neutron beam in time) is synchronous with the proton pulse in order to cut the fast neutron part of the spectrum, letting through only the thermal component. Choppers with this feature are commonly used at spallation sources, often known as TO choppers, referring to the fact that they effectively close the beam path at the moment of neutron production "time equal zero", or also as NIMONIC choppers, referring to the nickel alloy used as bulk material to intercept the fast neutrons [33]. The phase of these choppers can be changed in order to let the full spectrum through when necessary.

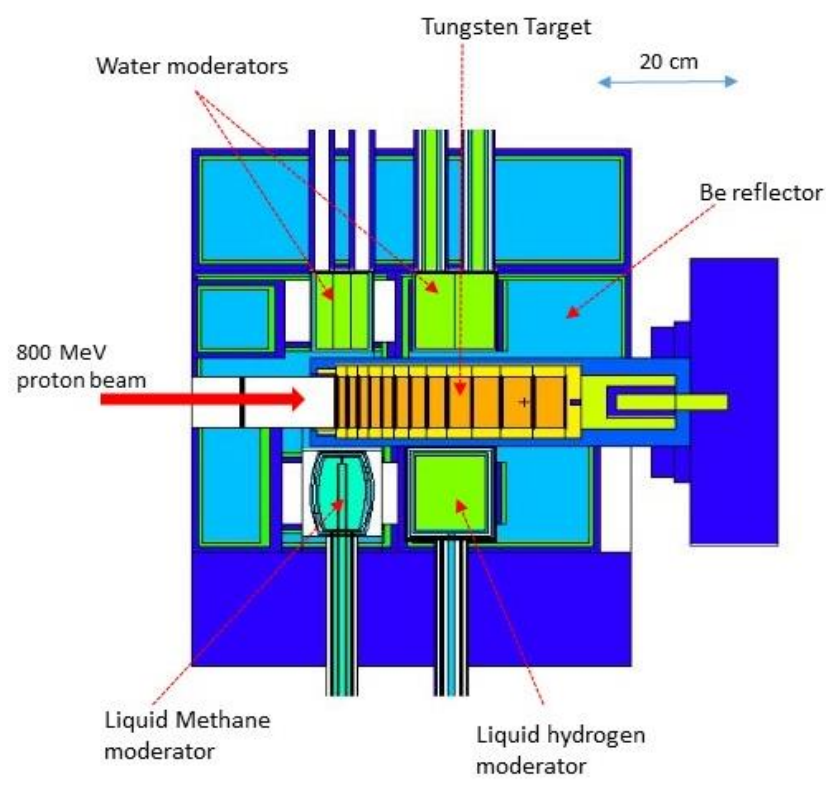

Fig. 2. Schematic of the ISIS first target station (vertical cut). The essential components of the target station are i) the tungsten target, ii) the moderators, iii) the beryllium reflector. Moderators of different materials are used to extract thermal neutrons at different temperatures. 


\section{GEM DETECTORS USING TIME OF FLIGHT TECHNIQUE}

In this work, Gas Electron Multiplier (GEM) detectors were used to measure flux and the spatial beam profiles. GEMs [22] are charged-particle detectors based on thin $(50 \mu \mathrm{m})$ insulating foils, cladded with copper on both sides. The charge multiplication is done in holes (diameter is about $70 \mu \mathrm{m}$ ) in the foils: when a bias voltage is applied to the copper layers each hole acts as a proportional amplifier [23].

To optimize the transport and collection of charges, the detector uses an atmosphere of $\mathrm{Ar}-\mathrm{CO}_{2}$, which is the standard mix used in triple GEM detectors. After multiplication, electrons are collected from the avalanche by electrodes.

Clearly, to detect thermal neutrons one needs to convert them to charged particles. To this purpose a thin layer of $\mathrm{B}_{4} \mathrm{C}$, where the boron is enriched ${ }^{10} \mathrm{~B}$ (enrichment $>99 \%$ as certified by the manufacturer), is deposited on the cathode, which is just below the entrance window [24, 25].

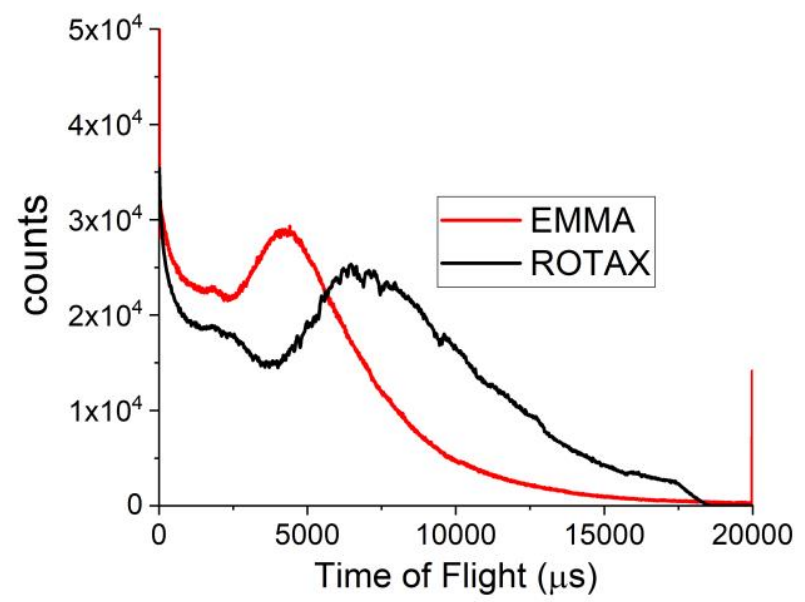

Fig. 3. Measurement of the Time of Flight spectra using a borated GEM detector.

Neutrons are counted when signals exceed the threshold of the readout electronics. The efficiency is characterized by the vendor and it is defined by the thickness $(\mathrm{t}=0.1 \mu \mathrm{m})$ of the thin layer of $\mathrm{B}_{4} \mathrm{C}$. The efficiency is a function of the neutron energy that scales as the cross section of ${ }^{10} \mathrm{~B}$ (see Fig. 1.). It is worth to notice that for a thin layer detector the efficiency at a given neutron energy $\varepsilon(E)$ can be approximated as $\varepsilon(\mathrm{E}) \approx \mathrm{n} \cdot \mathrm{t} \cdot \sigma_{10 \mathrm{~B}}(\mathrm{E})$ [32], where $\mathrm{n}$ is the number of ${ }^{10} \mathrm{~B}$ nuclei per unit volume, $\mathrm{t}$ is the thickness, and $\sigma_{10 \mathrm{~B}}$ is the capture cross section of ${ }^{10} \mathrm{~B}$.

A detector with a $10 \times 10 \mathrm{~cm}^{2}$ area and with a spatial resolution of $0.8 \mathrm{~mm}$ has been used.
A ToF histogram is built using the time difference between the $\mathrm{T}_{\text {start }}$ signal, coming from the proton on target pulse, and the detection $\mathrm{T}_{\text {stop }}$ signal. Fig. 3 shows counts as a function of ToF measured by the GEM detector on the two beamlines.

The energy of the neutron is easily calculated using classical kinematics

$$
E=\frac{1}{2} m\left(\frac{L}{T o F}\right)^{2}
$$

where $\mathrm{L}$ is the length of the neutron flight path to the detector and $\mathrm{m}$ is the mass of the neutron.

The energy spectrum $\frac{d N}{d E}$ is calculated from the ToF spectrum $\frac{d N}{d t}$ as

$$
\frac{d N}{d E}=\frac{d N}{d t}\left(\frac{\partial t}{\partial E}\right)
$$

The resulting neutron energy spectra are presented in Fig. 4. The neutron efficiency and the detection area are used to normalize the counts.

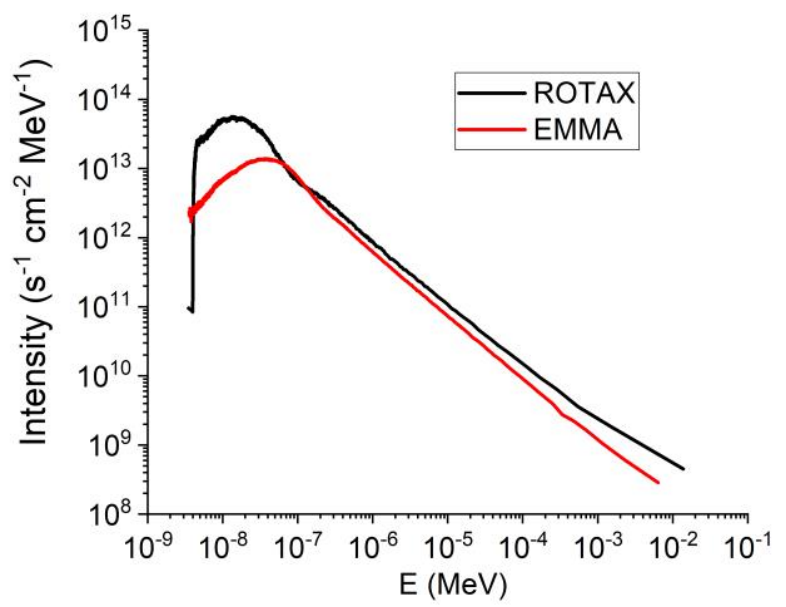

Fig. 4. Measured neutron energy spectra (dФ/dE) on two thermal beamlines at the ISIS pulsed neutron source. The beamlines have moderators of different temperature. 


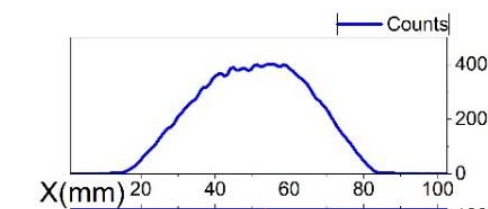

ROTAX
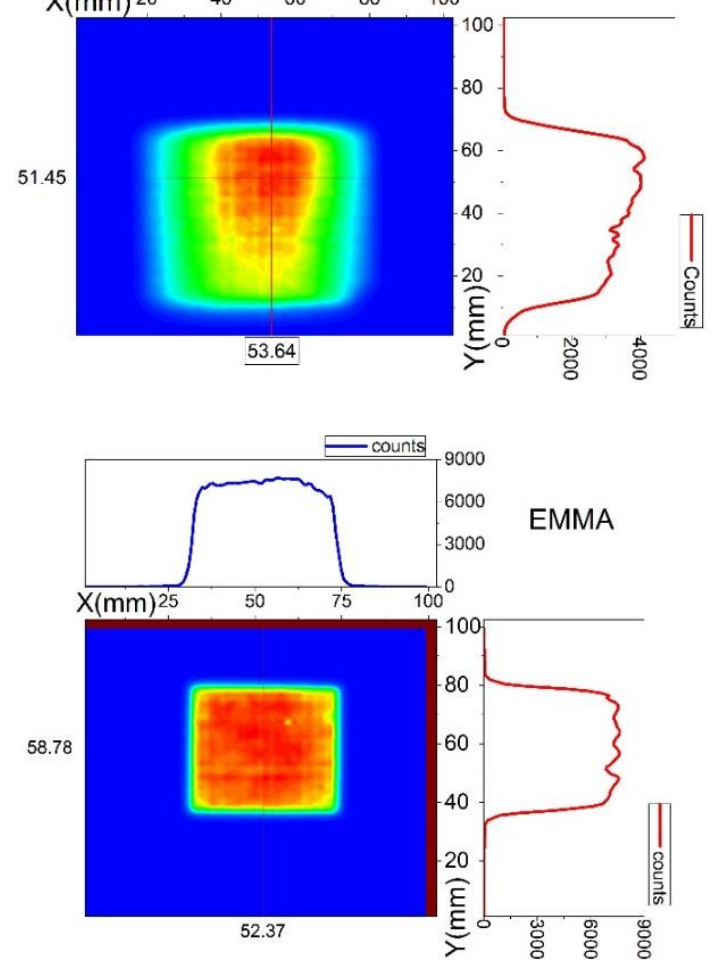

Fig. 5. Measurements of beam profiles on thermal neutron beamlines using a borated GEM detector. ROTAX (top) and EMMA (bottom).

It is very clear that the two beamlines have different thermal neutron spectra, mainly due to the different temperature of the moderators. Also the epithermal component presents a similar shape, where differences that can be attributed to the neutron transport. An important difference to be considered is that ROTAX has a forward direction, with respect to the proton beam, while EMMA is backwards.

The 2D spatial resolution of the detector allows for mapping of the beam profile, results are presented in Fig. 5. One can see that EMMA has a uniform square beam over the area of $4 \times 4 \mathrm{~cm}^{2}$, where standard deviation $\sigma /$ average $\mu=8 \%$. Different sizes could be selected using a $\mathrm{B}_{4} \mathrm{C}$ jaw system. ROTAX is less uniform, on an area of $4 \times 4 \mathrm{~cm}^{2}$, we find $\sigma / \mu=22 \%$, however a $2 \times 2 \mathrm{~cm}^{2}$ uniform area can be found, where $\sigma / \mu=6 \%$. On a ROTAX it is also possible to define smaller beams using $\mathrm{B}_{4} \mathrm{C}$ jaws.

In Fig. 6 it is possible to notice what happens to the spectrum of ROTAX and EMMA when the chopper is set in phase with the accelerator to cut the epithermal and fast component of the beam. One can see that the intensity for $\mathrm{E}_{\mathrm{n}}>1 \mathrm{eV}$ diminishes by about two orders of magnitude. These settings can be used when it is important to discard epithermal neutrons. When the chopper is set "out of frame" the entire spectrum can go through, except a small portion of cold neutrons that are very slow compared to the others and are still intercepted by the out of phase chopper.
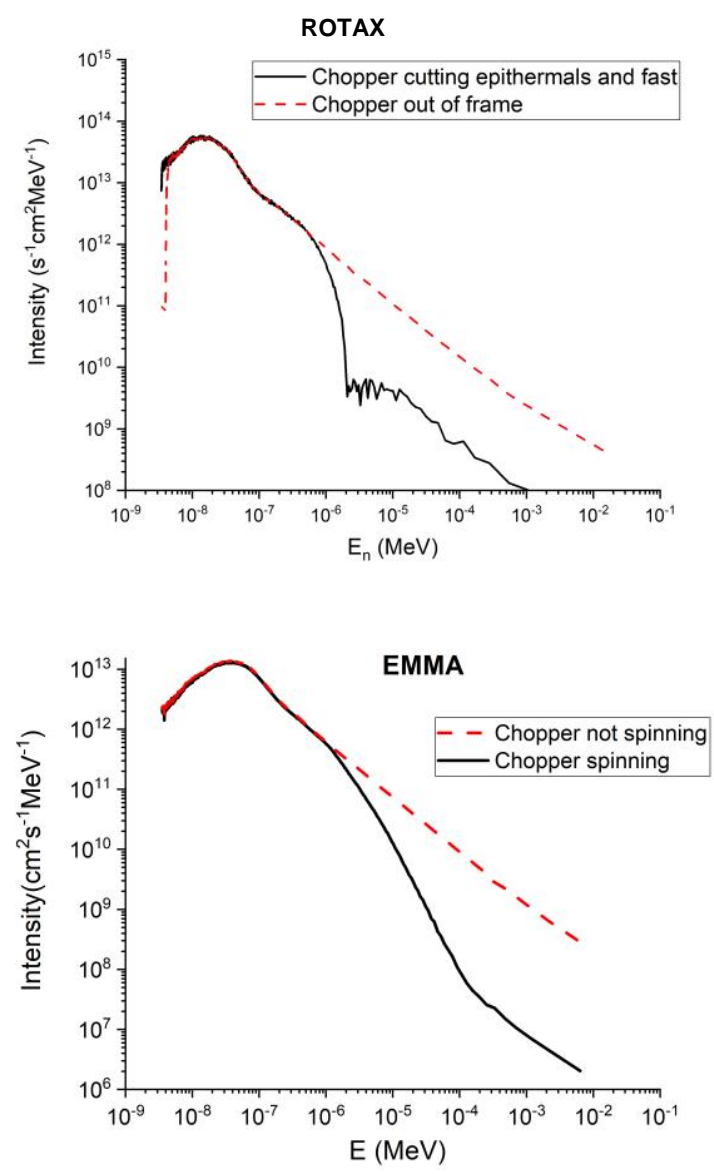

Fig. 6. Measured neutron energy spectra at ROTAX with the chopper spinning with different phase with respect to the proton pulse (top). Measured neutron energy spectra at EMMA with the chopper spinning and not spinning (bottom). The chopper can be used to cut the epithermal and fast component.

\section{ACTIVATION FOILS}

The neutron activation technique consists of irradiating foils of known material, i.e. containing a known amount of target nuclei $N^{\prime}$, and measuring the activation rate $R$. $R$ is the number of radio-nuclei produced in the sample per unit time during irradiation. This is given by the following equation

$$
R=N^{\prime} \Phi \int \sigma(E) \varphi(E) d E
$$

where $\Phi$ is the neutron flux, $\varphi(\mathrm{E})$ is the neutron spectrum normalized to the area $\frac{d N}{d E}=\Phi \cdot \varphi(E)$, and $\sigma$ is the 
nuclear cross section of the specific activation reaction. In this study, $\varphi(\mathrm{E})$ is based on the measurements by the GEM detector.

The measurement is performed with a High Purity Germanium detector (HPGe) that measures the $\gamma$-rays from the radioactive decay of product nuclei.

To infer $R$ from the measurement, one needs to count the number of decays $n_{\text {dec }}$ of the radio-nuclei after the irradiation. This is given by the number of counts $n_{\text {count }}$ in the signature peak divided by the efficiency, $\varepsilon$, and the probability of emission of a $\gamma$-ray of such energy, $I_{g}$.

$$
n_{\text {dec }}=\frac{n_{\text {count }}}{\varepsilon \cdot I_{g}}
$$

A coaxial detector system with a crystal of $70 \mathrm{~mm}$ diameter and $50 \mathrm{~mm}$ height has been used. Due to the large volume and small band gap, HPGe is the best solution in terms of efficiency and energy resolution. The efficiency has been calculated with a Monte Carlo simulation and benchmarked with laboratory calibration sources of ${ }^{60} \mathrm{Co},{ }^{137} \mathrm{Cs}$ and ${ }^{241} \mathrm{Am}$. These sources have $\gamma$ lines of known energy and the activity is certified by the manufacturer.
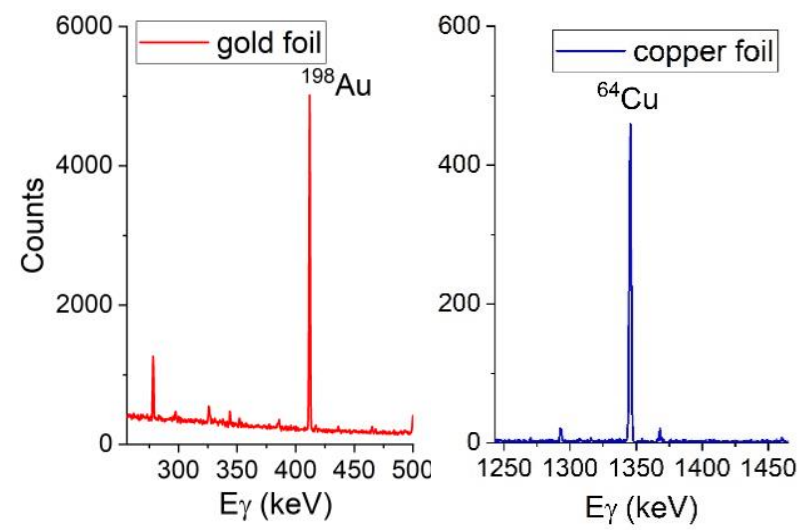

Fig. 7. Example of gamma-ray spectra measured by the HPGe after the activation of gold and copper foils on ROTAX.

The two reactions that have been selected for the activation measurements are ${ }^{197} \mathrm{Au}(\mathrm{n}, \gamma){ }^{198} \mathrm{Au}$ and ${ }^{63} \mathrm{Cu}(\mathrm{n}, \gamma){ }^{64} \mathrm{Cu}$. They are standard reactions for thermal neutron measurements. Product nuclei, ${ }^{198} \mathrm{Au}$ and ${ }^{64} \mathrm{Cu}$, have relatively long half-life, 2.69 days and 12.7 hours, respectively. Half-lives of days or hours are particularly favorable for the practicality of the measurement and are an important parameter in the choice of the isotopes. ${ }^{198} \mathrm{Au}$ and ${ }^{64} \mathrm{Cu}$ emit characteristic $\gamma$-rays at $411.8 \mathrm{keV}$ and $1345 \mathrm{keV}$, respectively. Irradiations of foils has been performed on both beamlines with the choppers in normal operation, spinning in phase with the beam, reducing epithermal and fast neutron fluxes (see Fig. 6). The ISIS accelerator was running at $160 \mu \mathrm{A}$ on target. The measurements of the $\gamma$-ray spectra are shown in Fig. 7, where the characteristic peaks are clear.

Results of flux measurements are shown in Table 1 where they are also compared to the thermal flux measured with the GEM detector (after integrating from 0 to $0.5 \mathrm{eV}$ of the spectra in Fig.4).

A discussion about uncertainties is needed. The gamma spectroscopy measurements have been repeated three times for EMMA, both for the gold and copper foil. The same relative uncertainties have been applied also to ROTAX, where a single gamma spectroscopy measurement was taken. However, one needs to consider that other systematic errors may remain uncharacterized, e.g. errors on the HPGe efficiency, alignment errors, etc. On the other hand, the copper and gold capture cross sections are very well known, so the cross section uncertainty is not expected to be a significant contribution. The uncertainty on the GEM detector efficiency was not given by the manufacturer. However, we can use a value of $10 \%$ as in Ref.[32], where a very similar GEM detector is presented. Taking into account these known uncertainties, Table 1 presents the weighted averages.

The measured neutron flux between the two techniques are in relative good agreement. For ROTAX all measurements are within 7\%, which is within the known uncertainties. For EMMA there is a discrepancy of about $19 \%$ between $\mathrm{Au}$ and $\mathrm{Cu}$ activation results, and a possible reason is discussed below following an analysis of the epithermal contribution. The values obtained from $\mathrm{Au}$ and $\mathrm{Cu}$ however give an average that has a discrepancy of only $4 \%$ with respect to the result given by the GEM detector. This is why two different reactions are used to check reproducibility of the results and average out possible systematic errors.

\begin{tabular}{c|cc|c} 
& gold & copper & Average \\
\hline EMMA & $(1.95 \pm 0.03) \cdot 10^{6}$ & $(2.34 \pm 0.14) \cdot 10^{6}$ & $(2.02 \pm 0.05) \cdot 10^{6}$ \\
ROTAX & $(2.90 \pm 0.04) \cdot 10^{6}$ & $(2.68 \pm 0.14) \cdot 10^{6}$ & $(2.85 \pm 0.06) \cdot 10^{6}$
\end{tabular}

\begin{tabular}{c|cc|c} 
& Activation & GEM detector & Average \\
\hline EMMA & $(2.02 \pm 0.05) \cdot 10^{6}$ & $(1.94 \pm 0.2) \cdot 10^{6}$ & $(2.0 \pm 0.1) \cdot 10^{6}$ \\
ROTAX & $(2.85 \pm 0.06) \cdot 10^{6}$ & $(2.72 \pm 0.3) \cdot 10^{6}$ & $(2.82 \pm 0.1) \cdot 10^{6}$
\end{tabular}

Table 1. Thermal neutron flux $(\mathrm{E}<0.5 \mathrm{eV})$ summary of measurements. Units are $\mathrm{cm}^{-2} \mathrm{~s}^{-1}$.

$\mathrm{Au}$ and $\mathrm{Cu}$ nuclear reactions are not covering the energy range with the same weight. This can be appreciated looking at the nuclear cross sections depicted in Fig. 8. 


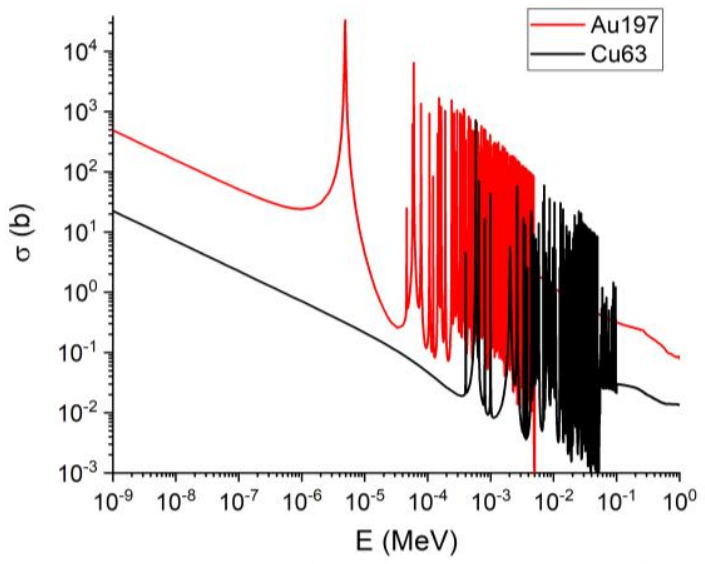

Fig. 8. Neutron capture cross sections of gold and copper as a function of the neutron energy.

In particular, gold has a strong resonance at about $5 \mathrm{eV}$, meaning that more weight is given to epithermal neutron then to thermal neutrons, with respect to copper. These have been taken into account by the analysis, as the activation rate for both is calculated using eq.3, integrating from $10^{-3} \mathrm{eV}$ to $10^{4} \mathrm{eV}$. In other words, even if in Table 1 we present only the neutron flux with $\mathrm{E}<0.5$ $\mathrm{eV}$, the flux above $0.5 \mathrm{eV}$ contributes to the analysis.

In Fig. 9 we present a breakdown of this analysis, where we calculated the contribution to the activation rate by three different energy groups: a) $\mathrm{E}<0.5 \mathrm{eV}$, b) $0.5 \mathrm{eV}<$ $\mathrm{E}<10 \mathrm{eV}$, c) $10 \mathrm{eV}<\mathrm{E}<10 \mathrm{keV}$.

We can notice the following facts:

- As expected due to the resonance, the contribution of group b) is always more for gold with respect to copper.

- On ROTAX, group a) is the main contribution for both gold and copper. On EMMA, group a) is the main contribution for copper, while group b) is the main contribution for gold. This is due to the different spectra on the beamlines with the thermal component on ROTAX being "colder" (where capture cross sections are higher).

- $\quad$ Energy group c), above $10 \mathrm{eV}$, contributes less than $1 \%$ both on ROTAX and on EMMA.

The second point can possibly explain why on EMMA there is more discrepancy between gold and copper results. This statement is based on an assumption that the difference is based on possible systematic errors in the spectra used at epithermal energies (given that the assumed spectra shape comes from GEM measurements). This analysis can be extended in future studies adding more measurements data. Firstly, activation measurements of foils embedded in $\mathrm{Cd}$ foil activation can be used to subtract the component coming from $\mathrm{E}>0.5 \mathrm{eV}$. Also more elements can be used and therefore more reactions with different resonances and thresholds. Having many reactions (with and without cadmium shielding) one can try a process of unfolding of the neutron spectrum, instead of relying, like here, on the guess spectrum measured by the GEM detector. However, this investigation is expensive in terms of both beam time and data analysis time. We argue that the current results are enough to enable the use of these beamlines for testing of microelectronics, as the testing community is increasing the demand of available beam time.
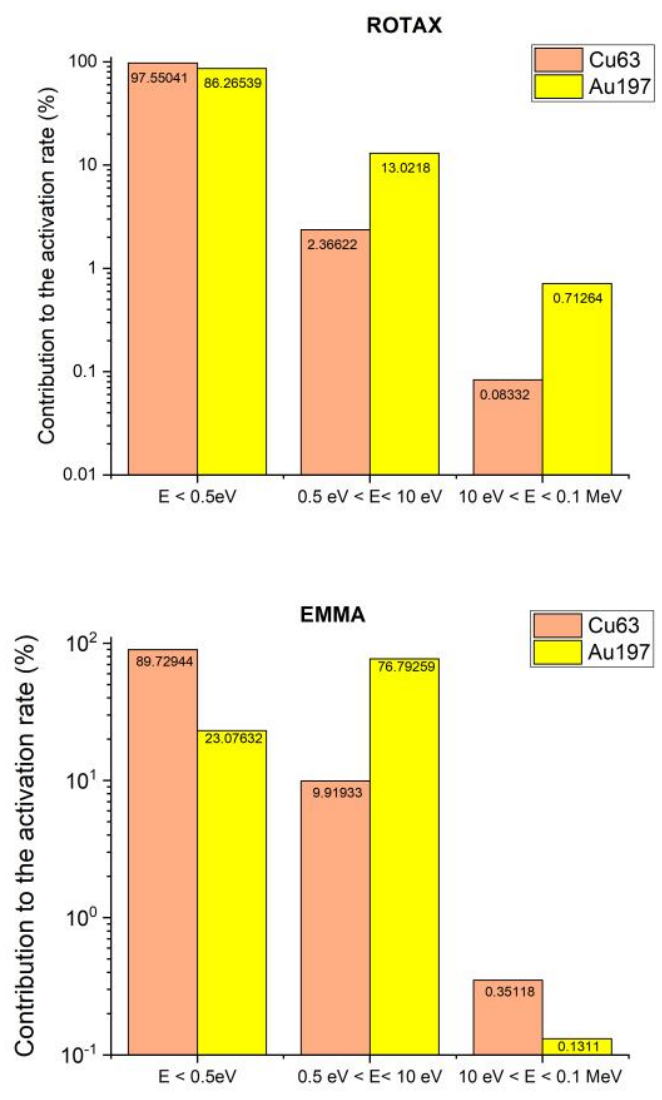

Fig. 9. Contribution to the activation rates of gold and copper for ROTAX (top) and EMMA (bottom).

\section{V.SEE MEASUREMENTS WITH SRAMS}

Test measurements have been performed using a detector based on SEEs. This is a reference monitor developed by the European Space Agency (ESA) [26]. It has been used to characterize the radiation field of space applications [27] and of different radiation facilities [28-31]. The ESA monitor is based on the detection of SEEs occurring in a set of 4 ICs of a well calibrated 4 Mbit SRAM (Atmel AT60142F), for a total of $16 \mathrm{Mbit}$. The die area of each chip has a size of $6.1 \mathrm{~mm} \times 11.2 \mathrm{~mm}$.

A check pattern of " 0 " and " 1 " is written in the memory before the start of the irradiation. After the device has been exposed to the neutron beam, the data are read and 
the number of errors are determined. One needs to be sure that the number of errors is small with respect to the 16 Mbit, so that the probability of the same bit being affected twice is negligible.

Measurements on the two beamlines have been performed, with and without a cadmium filter (see table 2). These measurements demonstrate that most of the SEE are indeed induced by thermal neutrons ( $88 \%$ and $97 \%$, respectively) and only a small percentage of SEE is induced by epithermals or residual fast neutrons. For this reason, users are always encouraged to perform control measurements with cadmium filters at thermal beamlines. The measurements on EMMA were performed with the accelerator operating at $175 \mu \mathrm{A}$, that is with respect to the measurements on ROTAX and all previous measurements, when the accelerator was operating at 160 $\mu \mathrm{A}$. The fluence has been corrected assuming that the flux scales linearly with the current of the accelerator. In order

\begin{tabular}{|c|c|c|c|c|c|c|c|}
\hline EMMA & SEEs & time(s) & $\mathrm{SEE} / \mathrm{s}$ & ROTAX & SEES & time(s) & $\mathrm{SEE} / \mathrm{s}$ \\
\hline No Cd & 1258 & 48944 & 0.0257 & No Cd & 2369 & 55140 & 0.043 \\
\hline $\mathrm{Cd}$ & 237 & 77802 & 0.003 & $\mathrm{Cd}$ & 31 & 21582 & 0.0014 \\
\hline
\end{tabular}

Table 2. Summary of the measurements performed with the ESA monitor on thermal beamlines. These are done with cadmium filter $(\mathrm{Cd})$ and without (No $\mathrm{Cd})$.

to calculate the thermal neutron cross section of the device at room temperature $(300 \mathrm{~K})$ the data of the EMMA beamline (moderated by water at $300 \mathrm{~K}$ ) can be directly used. On the other hand, one needs to apply a correction factor in order to use the data measured on ROTAX, because the moderator is significantly colder. This correction factor is given by the ratio of the convolution of the spectra and the nuclear cross section of ${ }^{10} \mathrm{~B}(\mathrm{n}, \alpha)^{7} \mathrm{Li}$, $\sigma_{10 \mathrm{~B}}$.

$$
\mathrm{C}=\frac{\int \sigma_{10 \mathrm{~B}}(\mathrm{E}) \varphi_{\text {RоT } A X}(\mathrm{E}) \mathrm{dE}}{\int \sigma_{10 \mathrm{~B}}(\mathrm{E}) \varphi_{\text {EMMA }}(\mathrm{E}) \mathrm{dE}}=1.69 \pm 0.05
$$

All irradiations were performed with the chopper spinning (see spectra of Fig.6) as this is the configuration most suitable for thermal neutrons.

An equivalent room temperature flux can be defined as

$$
\Phi_{\mathrm{eq}}=\frac{\Phi_{\mathrm{rotax}}}{C}
$$

After applying this correction, the results of the two beamlines can be compared (table 3). The results present a discrepancy of $15 \%$, which is not negligible but it

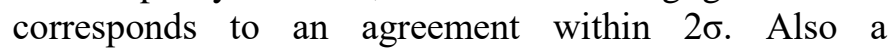

discrepancy on the order of $10 \%$ is not uncommon for test engineers testing the same component at different neutron facilities, and often considered a good result. However, to understand this discrepancy, uncertainties need to be further investigated.

The easiest contribution to evaluate is that of counting statistics, which given the numbers of Table 2 is on the order of 5\% (6\% for EMMA and 4\% for ROTAX).

The fluxes used in the analysis are the average of GEM and activation, as from the last column of Table 1.

The spectra used in the analysis are derived from the GEM-detector measurements, which implies that we truncate the integral at an upper bound of $\sim 10 \mathrm{keV}$ energy. It is reasonable to try to evaluate the error caused by this truncation. To this aim, we extend the same calculation to higher energy, assuming (as a worst case scenario) that

\begin{tabular}{l|ll} 
& EMMA & ROTAX \\
\hline $\begin{array}{l}\text { Thermal SEE } \\
\text { cross section at } \\
300 \mathrm{~K}\left(\mathrm{~cm}^{2}\right)\end{array}$ & $(1.03 \pm 0.06) \cdot 10^{-8}$ & $(0.87 \pm 0.05) \cdot 10^{-8}$ \\
& &
\end{tabular}

Table 3. Evaluation of the thermal SEE cross section at $300 \mathrm{~K}$.

the spectrum remains flat for $\mathrm{E}>10 \mathrm{keV}, \varphi(\mathrm{E})=$ $\varphi(10 \mathrm{keV})$. The result extending to $1 \mathrm{MeV}$ is a correction to $\mathrm{C}$ of $0.6 \%$. The result extending to $10 \mathrm{MeV}$ in the same way gives an uncertainty to $\mathrm{C}$ of $2.3 \%$.

It is also reasonable to try to evaluate the effect if there were a poor knowledge of the epithermal part of the spectrum. For this exercise, we multiply the epithermal flux of ROTAX (in the range $0.5 \mathrm{eV}-10 \mathrm{keV}$ ) by a factor of 3 and we notice that $\mathrm{C}$ changes only by $1.8 \%$. The reason is that $\sigma_{10 \mathrm{~B}}$ is decreasing with neutron energy, therefore, the main contribution is given by the low energy component.

The uncertainty from the knowledge of the ${ }^{10} \mathrm{~B}$ cross section (a well-known reference cross section) is about $0.2 \%$ in the thermal range, and $<1 \%$ in the range up to 90 $\mathrm{keV}$, according to evaluated nuclear data (ENDF/B-VII). These values are negligible for our analysis.

Combining the discussed uncertainties, we assume that an error of $3 \%$ is reasonable on the result of eq.5.

To obtain the uncertainties reported in Table 3, we combine only the counting statistics contribution and the correction factor contribution, giving an uncertainty of $6 \%$ for EMMA and of 5\% for ROTAX. The other main contribution is the uncertainties on the fluxes, on the order of 5\% (see Table 1). However, one needs to keep in mind that they are not statistically independent, as fluxes are measured with the same detectors. For this reason, we think it would be misleading to include this contribution in Table 3, when comparing the results. 
We suspect that another contribution to this discrepancy can be the non-uniformity of the ROTAX beam (see Fig.5) and the alignment of the board in the beam which could lead to an underestimate of the thermal SEE cross section. In fact the active area of the ESA monitor is $2 \times 2$ $\mathrm{cm}^{2}$. The beam of ROTAX has a standard deviation of $6 \%$ within the $2 \times 2 \mathrm{~cm}^{2}$, however this can increase up to $22 \%$ on a $4 \times 4 \mathrm{~cm}^{2}$ (although the alignment is performed with a laser, centered in the uniform $2 \times 2 \mathrm{~cm}^{2}$, and we do not expect a misalignment of more than a couple of $\mathrm{mm}$ ).

\section{CONCLUSION AND DISCUSSION}

Neutron measurements have been performed with a GEM detector, using the ToF technique, and with activation foils. The two beamlines that have been characterized with the accelerator at $160 \mu \mathrm{A}$ on target, EMMA and ROTAX, have thermal fluxes of $(2.0 \pm 0.1) \cdot 10^{6} \mathrm{~s}^{-1} \mathrm{~cm}^{-2}$ and $(2.8 \pm 0.1) \cdot 10^{6} \mathrm{~s}^{-1} \mathrm{~cm}^{-2}$, respectively. As expected, different thermal neutron spectra have been found due to moderators at different temperatures $(300 \mathrm{~K}$ vs. $100 \mathrm{~K})$. The two techniques, the active detector and the passive foils, give neutron fluxes that are relatively in good agreement. Discussions of the uncertainties and possible reasons for the discrepancies are presented.

The beamlines have then been used for SEE testing using a SRAM monitor, and results have been compared. The EMMA beamline is preferable because the beam profile is more uniform on a larger area of $4 \times 4 \mathrm{~cm}^{2}$, while the beam of ROTAX is uniform only within a smaller area of about $2 \times 2 \mathrm{~cm}^{2}$. We also notice that the moderator of EMMA, at room temperature $(300 \mathrm{~K})$, already matches most applications, without needing corrections. However, this does not mean that it is a better predictor of the component response, because, as it is shown, a spectrum from a colder moderator can be a valid alternative when a correction factor is applied. An advantage of ROTAX is the slightly higher flux, together with the boost in count rate (almost a factor of 2 ), given by the large cross section at lower temperature.

\section{REFERENCES}

[1] E. Normand, "Single-event effects in avionics," IEEE Trans. Nucl. Sci., vol. 43, no. 2, pp. 461-474, Apr. 1996.

[2] R. C. Baumann, "Radiation-induced soft errors in advanced semiconductor technologies," IEEE Transactions on Device and materials reliability 5(3) p.305 Sep. 2005.

[3] G. Russell, E. Pitcher, L. Daemen, "Introduction to spallation physics and spallation target design," AIP Conference Proceedings, Vol. 346 , AIP, 1995, pp. 93-104.

[4] M. S. Gordon et al., "Measurement of the Flux and Energy Spectrum of Cosmic-Ray Induced Neutrons on the Ground," IEEE Transactions on Nuclear Science 51 (6) 3427 (2004).

[5] C. Cazzaniga and C. D. Frost, "Progress of the Scientific Commissioning of a fast neutron beamline for Chip Irradiation," Journal of Physics: Conference Series, vol. 1021, p. 012037, May 2018

[6] M. Cecchetto et al., "SEE flux and spectral hardness calibration of neutron spallation and mixed field facilities," IEEE Transactions on Nuclear Science, vol. 66, no. 7, pp. 1532-1540, Jul. 2019.
S. A. Wender, "Neutron-Induced Failures in semiconductor Devices," Los Alamos National Laboratory, Los Alamos, NM, USA, Tech. Rep. LA-UR-17-22103, 2017.

[8] E.W. Blackmore, "Development of a large area neutron beam for system testing at TRIUMF," Radiation Effects Data Workshop, 2009 IEEE, IEEE, 2009, pp. 157-160.

[9] J. Dirk et al., "Terrestrial thermal neutrons," IEEE Transactions on Nuclear Science, vol. 50, no. 6, pp. 2060-2064, 2003.

[10] E. C. Auden et al., "Thermal neutron-induced single-event upsets in microcontrollers containing boron-10." IEEE Transactions on Nuclear Science 67.1 (2019): 29-37.

[11] R. C. Baumann and E. B. Smith. "Neutron-induced 10B fission as a major source of soft errors in high density SRAMs," Microelectronics Reliability 41.2 (2001): 211-218.

[12] D. Oliveira et al., "High-Energy Versus Thermal Neutron Contribution to Processor and Memory Error Rates," in IEEE Transactions on Nuclear Science, vol. 67, no. 6, pp. 1161-1168, June 2020.

[13] D. Oliveira et al., "Thermal Neutrons: a Possible Threat for Supercomputers and Safety Critical Applications," 2020 IEEE European Test Symposium (ETS), Tallinn, Estonia, 2020, pp. 1-6.

[14] L. Xu et al., "Thermal Neutron Induced Soft Errors in 7-nm Bulk FinFET Node," 2020 IEEE International Reliability Physics Symposium (IRPS), Dallas, TX, USA, 2020, pp. 616-620.

[15] J. M. Carpenter, "Pulsed spallation neutron sources for slow neutron scattering." Nuclear Instruments and Methods 145.1 (1977): 91-113.

[16] W. Kockelmann et al., "Energy-selective neutron transmission imaging at a pulsed source." Nuclear Instruments and Methods in Physics Research Section A: Accelerators, Spectrometers, Detectors and Associated Equipment 578.2 (2007): 421-434.

[17] W. N.McElroy et al., "Neutron-flux spectral determination by foil activation." Nuclear Science and Engineering 27.3 (1967): 533-541.

[18] D. Chiesa et al., "Measurement of the neutron flux at spallation sources using multi-foil activation." Nuclear Instruments and Methods in Physics Research Section A: Accelerators, Spectrometers, Detectors and Associated Equipment 902 (2018): 14-24.

[19] J. W. G. Thomason, "The ISIS spallation neutron and muon sourcethe first thirty-three years." Nuclear Instruments and Methods in Physics Research Section A: Accelerators, Spectrometers, Detectors and Associated Equipment 917 (2019): 61-67.

[20] G. P. Škoro and S. Ansell. "Neutronics modelling for the ISIS TS-1 upgrade," In Proceedings of the 21st Meeting of the International Collaboration on Advanced Neutron Sources ICANS XXI, Japan Atomic Energy Agency, pp. 141-147. 2016.

[21] W. Schäfer et al., "Setup and use of the ROTAX instrument at ISIS as angle-dispersive neutron powder and texture diffractometer," Nuclear Instruments and Methods in Physics Research Section A: Accelerators, Spectrometers, Detectors and Associated Equipment 364.1 (1995): 179-185.

[22] F. Sauli, "GEM: A new concept for electron amplification in gas detectors," Nuclear Instruments and Methods in Physics Research Section A: Accelerators, Spectrometers, Detectors and Associated Equipment 386.2-3 (1997): 531-534.

[23] S. Bachmann et al., "Charge amplification and transfer processes in the gas electron multiplier," Nuclear Instruments and Methods in Physics Research Section A: Accelerators, Spectrometers, Detectors and Associated Equipment 438.2-3 (1999): 376-408.

[24] G. Croci et al., "GEM-based thermal neutron beam monitors for spallation sources," Nuclear Instruments and Methods in Physics Research Section A: Accelerators, Spectrometers, Detectors and Associated Equipment 732 (2013): 217-220.

[25] G. Croci et al., "Characterization of a thermal neutron beam monitor based on gas electron multiplier technology," Progress of Theoretical and Experimental Physics 2014.8 (2014): 083H01.

[26] R. Harboe-Sørensen, F.-X. Guerre, and A. Roseng, "Design, testing and calibration of a reference SEU monitor system," in Proc. RADECS, 2005, pp. B3-1-B3-7.

[27] R. Harboe-Sørensen et al., "PROBA-II technology demonstration module in-flight data analysis," IEEE Transactions on Nuclear Science 59.4 (2012): 1086-1091.

[28] R. García Alía et al., "SEU measurements and simulations in a mixed field environment," IEEE Transactions on Nuclear Science 60.4 (2013): 2469-2476.

[29] C. Cazzaniga et al., "Atmospheric-Like Neutron Attenuation During Accelerated Neutron Testing With Multiple Printed Circuit Boards," IEEE Transactions on Nuclear Science 65.8 (2018): 1830-1834. 
[30] M. Cecchetto et al., "Impact of thermal and intermediate energy neutrons on SRAM SEE rates in the LHC accelerator," IEEE Transactions on Nuclear Science 65.8 (2018): 1800-1806.

[31] M. Kastriotou et al., "Single Event Effect Testing With Ultrahigh Energy Heavy Ion Beams," IEEE Transactions on Nuclear Science 67.1 (2019): 63-70.

[32] A. Muraro et al.,"Performance of the high-efficiency thermal neutron BAND-GEM detector." Progress of Theoretical and Experimental Physics 2018.2 (2018): 023H01.

[33] S. Itoh et al., "T0 chopper developed at KEK." Nuclear Instruments and Methods in Physics Research Section A: Accelerators, Spectrometers, Detectors and Associated Equipment 661.1 (2012): 8692. 